\title{
Computer-Aided Porous Materials Description Based on Morphological Spectra
}

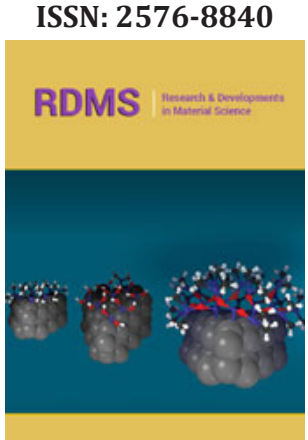

*Corresponding author: Juliusz L Kulikowski, Nalecz Institute of Biocybernetics and Biomedical Engineering PAS, Warsaw, Poland

Submission: 诲 August 30, 2019

Published: 㘹September 10, 2019

Volume 11 - Issue 4

How to cite this article: Juliusz Kulikowski. Computer-Aided Porous Materials Description Based on Morphological Spectra. Res Dev Material Sci. 11(4).RDMS.000769.2019.

DOI: 10.31031/RDMS.2019.11.000769

Copyright@ Juliusz L Kulikowski, This article is distributed under the terms of the Creative Commons Attribution 4.0 International License, which permits unrestricted use and redistribution provided that the original author and source are credited.

\section{Juliusz L Kulikowski*}

Nalecz Institute of Biocybernetics and Biomedical Engineering PAS, Poland

\section{Summary}

A new approach to porous materials features evaluation is proposed in the paper. The method consists in computer analysis of microscope images of porous material sections. Instead of individual pores analysis, in this approach global morphological spectra (MS) of the images are examined. It is shown that MS contain full information about the morphological structure of the porous material section. Porosity evaluation is then formulated as a statistical MS classification task and a method of its solution is proposed.

Keywords: Porous materials; Porosity evaluation; Morphological spectra; Computer-aided image analysis

\section{Introduction}

Synthetic porous materials, e.g. based on polyamide, polyether sulfone, polyvinylpyrrolidone etc. compounds, have numerous and important medical applications. They are used as filtering membranes in hemodialysis [1], micro-capsules for cells or drugs encapsulation and delivery [2], scaffolds for biological tissue cultivation [3], etc. Usefulness of porous materials in medical applications directly depends on their biochemical and mechanical properties, in particular, on porosity characteristics. The last should thus be carefully controlled within a production process and keptstable for exploitation period. For this purpose, numerical parameters describing size and shape of pores as well as global material porosity characteristics (porosity factor, material's permeability, multi-layer architecture of porous membranes etc.) have been proposed [4,5]. In fact, the parameters don't describe the pores directly but their planar cross-sections visible in SEM (Scanning Electron Microscope) images. Moreover, such geometrical parameters like pore section's spread, surface, length of contour etc. are by computer systems interpreted in a discrete geometry sense, not quite consistent with the widely known Euclidean geometry. The differences are particularly clear in case of small (consisting of several pixels) geometrical objects analysis.

Figure 1 shows z typical SEM image of a cross-section of a sample of porous material: $a$ ) in original form and $b$ ) after image enhancement procedures, visualizing selected (deep) pores as white objects.

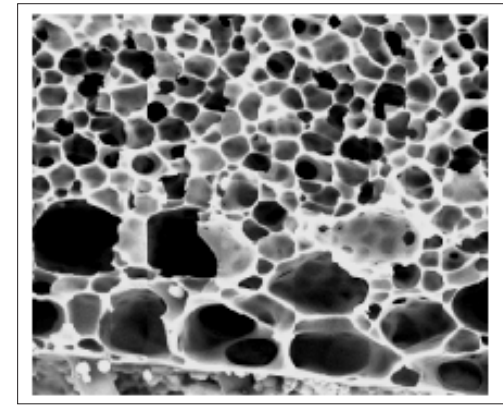

a)

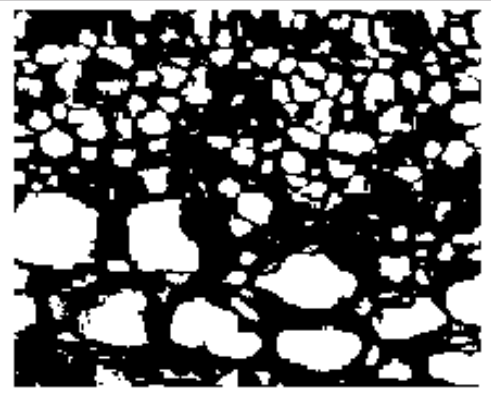

b)
Figure 1: SEM image of a sample of porous material: a) original, b) prepared for computer analysis.

The image in the transformed and enhanced form can then be subjected to next steps of computer analysis consisting of evaluation the size, extension and form of selected objects, their, statistical analysis etc. [4,5]. This work can be performed by commercial (like Matlab [6]) 
or more effective, specialized (like Memo Explorer [7]) computer programs. In any case, the calculation of geometrical parameters of pores is based on the results of counting pixels belonging to the considered objects or constituting their contours or inner areas. However, the number of pixels strongly depends on the results of preliminary image enhancement procedures. For example, in the case presented by Figure 1 some hollows have not been qualified as pores despite the fact that they may be shallow bottoms of cut away larger pores. In this paper an alternative approach to porous materials assessment is proposed. The approach is based on global analysis of SEM images of porous material cross-section without segmentation and analysis of individual pores. The approach is based on the assumption that both, spatial structure of a porous material and images of its planar cross-sections are some statistically dependent, respectively 3-dimensional and 2-dimensional, random fields. Porous materials should thus be evaluated and/or classified on the basis of their 3-dimensional statistical properties, available only through the instances of their observable 2-dimensional cross-sections. Mining information by extrapolation of available data is one of important artificial intelligence (AI) problems [8]; in this case it is limited to one, specific type of random objects. For global image description morphological spectra (MS), based on a modification of 2-dimensional Walsh functions are proposed $[9,10]$. The utility of $M S$ to description and analysis of textures was presented in $[11,12]$; in fact, the images of porous materials crosssections can be considered as textures as well. In the paper $M S$ of artificial patterns of porous materials cross-sections are presented. Porous materials characterization consists then in calculation of the $M S$ of its cross-section and in matching it to pattern MS simulating the previously established size- and/or shape-classes of porosity. Such approach works thus on whole images instead of analysis of individual pores. The paper is organized as follows: in Sec 2 basic information about $M S$ is shortly presented. Sec 3 contains examples of $M S$ of typical porous patterns. Principles of porosity evaluation based on higher order $M S$ are described in Sec 4. Conclusions are summarized in Sec 5.

\section{Basic information about morphological spectra}

Morphological spectra (more exactly, 2-dimensional morphological spectra, MS2) are the effect of expansion of a monochromatic image described on a discrete plane into a series of basic morphological functions $\Phi^{(r)}=\left\{\Phi_{m}\right\}$ of a fixed natural order $r$ [13]. The basic $\mathrm{r}^{\text {th }}$ order morphological functions, in some publications, due to their graphical interpretation called also masks, are binary modifications of discrete Walsh functions [10]. The set $\Phi^{(r)}$ consists of $2^{r}$ binary functions $\Phi_{\mu}$ described on a $2^{r} \times 2^{r}$ size discrete square:

$$
\begin{gathered}
\phi_{\mu}=\left[f_{\dot{a}, \hat{a}}^{(\mu)}\right](1) \\
\text { where } p, q \in\left[1, \ldots, 2^{r}\right], f_{\dot{a}, \hat{a}}^{(\mu)} \in[-1,1]
\end{gathered}
$$

For $r=1$ the functions have the form shown below in tabular form:

$$
\phi_{S}=\begin{array}{|l|l|}
\hline 1 & 1 \\
\hline 1 & 1 \\
\hline
\end{array}, \phi_{V}=\begin{array}{|l|l|}
\hline-1 & 1 \\
\hline-1 & 1 \\
\hline
\end{array}, \phi_{H}=\begin{array}{|l|l|}
\hline-1 & -1 \\
\hline 1 & 1 \\
\hline
\end{array}, \phi_{X}=\begin{array}{|l|l|}
\hline-1 & 1 \\
\hline 1 & -1 \\
\hline
\end{array}
$$

The indices $S, V, H$ and $X$, when written in bold, can be interpreted as symbols of operators used to generation of higher order basic functions, as it will be shown below. For $r=2$ we obtain $2^{2} \times 2^{2}=16$ $2^{\text {nd }}$ order basic functions denoted by $\varphi_{S S,} \varphi_{V S,} \varphi_{H S, \ldots,} \varphi_{X X}$, The functions are described on $4 \times 4$ discrete squares and can be generated by subjecting the lower-order functions $\varphi_{S_{S}} \varphi_{V,} \varphi_{H_{u}} \varphi_{X}$ to the operations $S, V, H$ or $X$ as illustrated by the following example:

\begin{tabular}{|c|c|c|c|c|c|c|}
\hline \multirow{2}{*}{$-\Phi_{H}$} & \multirow{2}{*}{$\Phi_{H}$} & \multirow{4}{*}{$=$} & 1 & 1 & -1 & -1 \\
\hline & & & -1 & -1 & 1 & 1 \\
\hline \multirow{2}{*}{$-\Phi_{H}$} & \multirow{2}{*}{$-\Phi_{H}$} & & 1 & 1 & -1 & -1 \\
\hline & & & -1 & -1 & $1 \mathrm{ss}$ & 1 \\
\hline
\end{tabular}

$$
V\left(\varphi_{H}\right) \equiv \varphi_{V H}(2)
$$

or in a more extended form:

In similar way, the higher, $r^{\text {th }}$ order basic morphological functions can be composed of the lower, $(r-1)^{\text {th }}$ order functions [12]. Increasing of the order $r$ by 1 causes extension of the working window size to $(r+1) \times(r+1)$ and a corresponding increasing of the number of the $M S$ components. Let $\boldsymbol{U}=\left[u_{i, j}\right]$ denote a digitalized form (a bitmap) of an $I \times J$ size monochromatic image consisting of pixels $u_{i, j} \in\left[0,1, \ldots, 2^{k}-1\right], k$ being a natural number characterizing the gradation of pixel value brightness. Additionally, it will be assumed that the numbers $I$ of rows and $J$ of columns of the image satisfy the conditions: $\mathrm{I}=\mathrm{k} \cdot 2^{\mathrm{r}}, \mathrm{J}=\mathrm{l} \cdot 2^{\mathrm{r}}, \mathrm{k}$ and $l$ being some natural numbers. Then $U$ can be partitioned into $k \times l$ sub-arrays:

$$
U=\left[\boldsymbol{U}^{(\hat{e}, \ddot{e})}\right](3)
$$

described on $2^{r} \times 2^{r}$ - size working windows. The sub-arrays have thus the form:

$$
\boldsymbol{U}^{(\hat{e}, \hat{e})}=\left[u_{i, j}^{(\hat{e}, \hat{e}}\right],(4)
$$

and the subsets of admissible indices $i, j$ is limited to those corresponding to the given sub-array.

For any $r^{\text {th }}$ order basic morphological function $\varphi_{\mu}$ and conformable to it (being of the same size) working sub-array $U^{(\kappa, \lambda)}$ their scalar product:

$$
w_{\hat{e}, \ddot{e}}^{(\mu)}=\boldsymbol{U}^{(\hat{e}, \ddot{e})} * \phi_{\mu}=\sum_{i, j} u_{i, j}^{(\hat{e}, \ddot{e})} \cdot f_{i, j}^{(\mu)}(5)
$$

can be calculated. The coefficient $w_{e, e}^{(\mu)}$ is called an $\mathrm{r}^{\text {th }}$ order $\mu^{\text {th }}$ MS component of $U^{(\kappa, \lambda)}$. The set $=\left[w_{\dot{e}, \bar{r}}^{(\mu)}\right]$ arranged in a $2^{r} \times 2^{r}$-size table of $M S$ components is called an $r^{\text {th }}$ order $M S$ of $U^{(\kappa, \lambda)}$. It should be remarked that due to a specific binary structure of the functions $\phi_{\mu}$ calculation of $W_{\kappa, \lambda}$ is reduced to adding and subtracting some combinations of the components $u_{i, j}^{i, g}$.

A table consisting of $\mathrm{r}^{\text {th }}$ order $\mu^{\text {th }} M S$ components of all $U^{(\kappa, \lambda)}$ :

$$
\boldsymbol{W}_{r}^{(\mu)}=\left[w_{\hat{e}, \tilde{e}}^{(\mu)}\right](6)
$$

is called a $\mathrm{r}^{\text {th }}$ order $\mu^{\text {th }} M S$ component of $\boldsymbol{U}$. For a fixed order $r$ there are $2^{r} \times 2^{r} \mu^{\text {th }} M S$ components of all $\boldsymbol{U}^{(\kappa, \lambda)}$, constituting a full $r^{\text {th }}$ order MS of $\boldsymbol{U}$ :

$$
\boldsymbol{W}_{r}=\left[\boldsymbol{W}_{r}^{(\mu)}\right]
$$


It can be shown that $W_{r}$ contains full information about $U$. Really, it consists totally, like $\mathrm{U}$, of $\mathrm{k} \cdot \mathrm{l} \cdot 2^{\mathrm{r}} \cdot 2^{\mathrm{r}}$ real components of $\boldsymbol{w}_{\hat{e}, \hat{e}}^{(\boldsymbol{\mu})}$ type and $U$ can be reconstructed if $W_{r}$ is given. The properties of $U$ are then hidden in the features of $W_{r^{*}}$

\section{MS of selected porosity patterns}

In this Section it will be shown that to some typical porosity patterns the corresponding morphological spectra can be assigned. For this purpose, exemplary porosity patterns in $2^{2} \times 2^{2}$-size working windows have been generated and for their characterization the $2^{\text {nd }}$ order $M S$ will be used. It also will be assumed that the porosity patterns in the form of binary images, as white pores $\left(u_{i, j}=1\right)$ on black background $\left(u_{i, j}=1\right)$ are presented. In a $4 \times 4$-size working window in general $2^{16}=65536$ different binary images can be described, only some of them presenting typical porosity patterns. For example, a class $C_{1}$ of porosity patterns consisting of 1 "white" pixel (corresponding to a small pore) within 15 "black" pixels (representing a background) contains 16 instances of porosity patterns. Below, for exemplary purposes, the following classes of porosity patterns will be taken into consideration:

A. $\quad \mathrm{C}_{0}$ : no pixels representing pores in a working window;

B. $\mathrm{C}_{1}: 1$ single pixel representing a pore in the working window;

C. $\quad \mathrm{C}_{1+1}: 2$ single pixels representing 2 separate pores in the working window;

D. $\quad \mathrm{C}_{2}: 2$ adjacent pixels representing 1 pore in the working window;

E. $\quad \mathrm{C}_{2+2}: 2$ pairs of adjacent pixels representing 2 separate pores in the working window.

In practice, if necessary, the number of classes can be increased. An object is called compact if it consists of more than 1 pixel and each of the pixels is adjacent to at least one other pixel of the object; the 8-connectivity of pixels (each central pixel surrounded by 8 adjacent pixels) on a rectangular greed is assumed. Pores, by definition, are represented by compact sets of white pixels. For description of the above-mentioned classes the following $2^{\text {nd }}$ order basic morphological functions will be used:

$$
\begin{gathered}
\Phi^{(2)}=\left\{\varphi_{S S^{\prime}} \varphi_{S V^{\prime}} \varphi_{S H^{\prime}} \varphi_{S X^{\prime}} \varphi_{V S^{\prime}} \varphi_{V V^{\prime}} \varphi_{V H^{\prime}} \varphi_{V X^{\prime}} \varphi_{H S^{\prime}} \varphi_{H V^{\prime}} \varphi_{H H^{\prime}} \varphi_{H X^{\prime}} \varphi_{X S^{\prime}}\right. \\
\left.\varphi_{X V^{\prime}}, \varphi_{X H^{\prime}} \varphi_{X X}\right\} \cdot(8)
\end{gathered}
$$

In case of higher image magnification, when the smallest pores by compact groups of up to 4 white pixels are presented, the set $\Phi^{(2)}$ can be replaced by a set $\Phi^{(3)}=\left\{\varphi_{S S S^{\prime}} \varphi_{S V S^{\prime}}, \ldots, \varphi_{X X S}\right\}$ of basic morphological functions described on the $2 \times 2$-size squares. According to formula (5), the following $2^{\text {nd }}$ order $M S$ components will then describe the classes of porosity patterns:

$$
\begin{gathered}
V^{(2)}=\left\{v_{S S^{\prime}} v_{S V^{\prime}} v_{S H^{\prime}} v_{S X^{\prime}} v_{V S^{\prime}} v_{V \nu^{\prime}}, v_{V H^{\prime}} v_{V X^{\prime}} v_{H S^{\prime}} v_{H V^{\prime}} v_{H H^{\prime}} v_{H X^{\prime}} v_{X S^{\prime}} v_{X V^{\prime}} v_{X H^{\prime}}\right. \\
\left.v_{X X}\right\} \cdot(9)
\end{gathered}
$$

For the above-defined porosity classes two groups of conditions satisfied by the corresponding $M S$ can be mentioned:

\section{a) Conditions determining the size of objects.}

The size of objects is described by the value of $v_{S S}$ Therefore, 1) $v_{S S}=0$ for $C_{0}$;2) $v_{S S}=1$ for $C_{1}$; 3) $v_{S S}=2$ for $C_{1+1}$ and $C_{2 ;}$ 4) $v_{S S}=4$ for $C_{2+2}$, etc.

b) Conditions determining the compactness of objects.

First, a hypothesis that morphological spectra of separate and compact pairs of pixels representing pores are different should be proven. For this purpose, all different instances of separate and compact pairs of white pixels in a $4 \times 4$-size working window have been generated and subjected to analysis. The number of the pairs of separate pixels equals 78 , the number of the compact pairs equals 41 . For the sake of simplicity the pixels in a $4 \times 4$-size working window will be addressed as shown in Table 1. Moreover, the indices $\kappa, \lambda$ identifying the working window in this Section will be omitted. We denote by $\Omega=\left\{\omega_{p, q}\right\}$ the set of pattern compositions of pairs of pixels in a working window; the indices $p, q$ denoting the addresses of "white" pixels. The set is a sum of disjoint subsets:

$$
\Omega=\Omega^{I} \cup \Omega^{I I}(10)
$$

Table 1: Addresses of pixels in a $2 \times 2$-size working window.

\begin{tabular}{|c|c|c|c|}
\hline 1 & 2 & 3 & 4 \\
\hline 5 & 6 & 7 & 8 \\
\hline 9 & 10 & 11 & 12 \\
\hline 13 & 14 & 15 & 16 \\
\hline
\end{tabular}

where $\Omega^{1}$ denotes a subset of patterns consisting of disjoint pixels (78 possible cases), $\Omega^{I I}$ stands for a subset of patterns consisting of adjacent pixels (41 possible cases). For example, in Figure 2 the patterns $\omega_{1,11}$ and $\omega_{5,7}$ belonging to $\Omega^{I}$ and $\omega_{6,7}$ and $\omega_{6,11}$ belonging to $\Omega^{I I}$ are shown. For the instances of images presenting typical pores the corresponding $M S$ can be calculated. The $M S_{2}$ components of the exemplary images shown in Figure 2 are given in Table 2. It is remarkable that the $M S_{2}$ components have the form of series composed of three values: $-2,0$ and 2 . The component $v_{S S}$ characterizes the size of pores. The components $v_{S V}, v_{S X}$ and $v_{X X}$ strongly discriminate the pairs of separate and adjacent pixels. On the other hand, the discriminating power of $v_{S H}$ and $v_{H H}$ seems to be rather negligible.
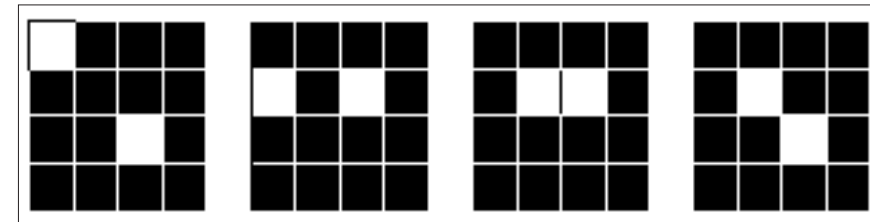

$\omega_{l, 11}$

$\omega_{5,7}$

$\omega_{6,7}$

$\omega_{6,11}$

Figure 2: Examples of pattern pixels allocation in a working window. 
Table 2: MS2 components' values of pairs of pixels' compositions shown in Figure 2.

\begin{tabular}{|c|c|c|c|c|c|c|c|c|c|c|c|c|c|c|c|c|}
\hline $\begin{array}{c}\text { MS } \\
\text { Pix. }\end{array}$ & $v_{s S}$ & $v_{S V}$ & $v_{S H}$ & $v_{s x}$ & $v_{v s}$ & $v_{v V}$ & $v_{V H}$ & $v_{V X}$ & $v_{H S}$ & $v_{H V}$ & $v_{\text {нн }}$ & $v_{H X}$ & $v_{X S}$ & $v_{X V}$ & $v_{X H}$ & $v_{X X}$ \\
\hline$[1,11]$ & 2 & -2 & 0 & 0 & -2 & 2 & 0 & 0 & 0 & 0 & 2 & -2 & 0 & 0 & 2 & -2 \\
\hline$[5,7]$ & 2 & -2 & 0 & 0 & 2 & -2 & 0 & 0 & -2 & 2 & 0 & 0 & -2 & 2 & 0 & 0 \\
\hline$[6,7]$ & 2 & 0 & 0 & -2 & 2 & 0 & 0 & -2 & -2 & 0 & 0 & 2 & -2 & 0 & 0 & 2 \\
\hline$[6,11]$ & 2 & 0 & 0 & -2 & 0 & 2 & -2 & 0 & 0 & -2 & 2 & 0 & -2 & 0 & 0 & 2 \\
\hline
\end{tabular}

The above-presented results have been related to only 2 (out of 78) pairs of separate and to 2 (out of 41) pairs of adjacent pixels. The problem thus arises of possible determination of the criteria for discrimination in a more general case the separate and adjacent pairs of pores presenting pixels. For this purpose, the percentage of the values of MS2 components' for all combinations of the pairs of "white" pixels have been calculated. The results are presented in Table 3. The signs of components with higher probability being

assigned to one of the alternative types of pairs are marked in bold. Assume that for the $U^{(\kappa, \lambda)}$ working sub-array its $M S 2$ according to formula (5) has been calculated. It consists of $4 \times 4=16$ components $w_{\hat{e}, \ddot{e}}^{(\mu)}$ constituting a vector (the indices $\kappa, \lambda$ for simplicity being neglected): $W_{k, \lambda}=\left[w^{(S S)}, w^{(S V)}, w^{(S H)}, \ldots, w^{(X H)}, w^{(X X)}\right]$. Assume, for instance, that the values (signs of values) of the components of $W_{k, \lambda}$ are as follows:

$$
W_{k, \lambda}=[2,+,+, 0,+,-, 0,+,+,-,-, 0,0,+,+,-] .
$$

Table 3: Percentage of positive, null and negative $M S 2$ components in the spectra of the objects of class $C_{1+1}$ or $C_{2}$.

\begin{tabular}{|c|c|c|c|c|c|c|c|c|c|c|c|c|c|c|c|c|c|}
\hline \multicolumn{2}{|c|}{$\begin{array}{c}\text { MS Comp } \\
\text { Class Values }\end{array}$} & $S S$ & $S V$ & $S H$ & $S X$ & $V S$ & $V V$ & $V H$ & $V X$ & HS & $H V$ & $H H$ & $H X$ & $X S$ & $X V$ & $X H$ & $X X$ \\
\hline \multirow{3}{*}{$C_{1+1}$} & + & 100 & 28 & 17 & 29 & 28 & 24 & 27 & 26 & 15 & 27 & 21 & 24 & 28 & 26 & 24 & 22 \\
\hline & 0 & 0 & 28 & 15 & 16 & 28 & 24 & 26 & 24 & 15 & 28 & 19 & 22 & 15 & 26 & 21 & 26 \\
\hline & - & 0 & 44 & 68 & 55 & 44 & 52 & 47 & 50 & 70 & 45 & 60 & 54 & 57 & 48 & 55 & 52 \\
\hline \multirow{3}{*}{$C_{2}$} & + & 100 & 12 & 39 & 15 & 15 & 22 & 17 & 20 & 39 & 17 & 29 & 22 & 15 & 20 & 20 & 22 \\
\hline & 0 & 0 & 15 & 37 & 36 & 15 & 22 & 17 & 20 & 37 & 15 & 32 & 24 & 39 & 20 & 20 & 22 \\
\hline & - & 0 & 73 & 24 & 49 & 70 & 56 & 66 & 60 & 24 & 68 & 39 & 54 & 46 & 60 & 60 & 56 \\
\hline
\end{tabular}

The interpretation of the above-given result can be as follows:

a) $\quad w^{(S S)}=2$, therefore, the object belongs to class $C_{1+1}$ or $C_{2}$;

b) According to Table 3 , the recommendations for assigning $W_{\kappa, \lambda}$ to $C_{1+1}$ or to $C_{2}$ are as presented in the Table 4 . Therefore, in most (10 of 15) cases the signs of the $W_{\kappa, \lambda}$ components suggest its belonging to the class $C_{2}$. The suggestion doesn't take into account exact numerical values of the components. However, if it is doubtful it can be verified by finding among the spectra $V^{(2}$ of pattern pore images the most to $W_{k, \lambda}$ similar one.

Table 4: Recommendations for classification of $\boldsymbol{W}_{\kappa, \lambda}$.

\begin{tabular}{|c|c|}
\hline Type of Recommendation & $\begin{array}{c}\text { MS2 Components Suggesting a Recom- } \\
\text { mendation }\end{array}$ \\
\hline$W_{\kappa, \lambda} \in C_{1+1}$ & $S V, V S, V H, V X, H H$ \\
\hline$W_{\kappa, \lambda} \in C_{2}$ & $S X, S H, V V, H S, H V, H X, X S, X V, X H, X X$ \\
\hline
\end{tabular}

\section{Porosity evaluation based on higher-order MS}

Description of large pores needs using adequately increased working windows and higher order $M S$. Standard sizes of working windows are $2 \times 2,4 \times 4,8 \times 8,16 \times 16$, etc. The corresponding numbers of $M S$ components are then $4,16,64,256$, etc. The ratio:

$$
\gamma_{\hat{e}, \ddot{e}}=\frac{w_{\hat{e}, \ddot{e}}^{(S)}}{2^{2 r}}
$$

can be used as a local porosity factor of a working window. A global porosity factor of a material then can be defined as an average of local porosity factors over the set of all working windows:

$$
g=A v_{(x, \lambda)}\left(\gamma_{x, \lambda}\right) \text {. (12) }
$$

Pores' shape classification based on evaluation of porous material section similarity to the sets of pore patterns (as illustrated in the former Section) in the case of large working windows seems to be rather unrealistic because of large numbers of pore shape instances. Assuming that each class $C_{\varepsilon}$ of pores as a random field is by its proper statistical characteristics described, the problem of pores classification can be formulated as a statistical pattern recognition problem [14]. For this purpose, a statistical model $M_{\varepsilon}$ of each class $C_{\varepsilon}$ should be established. In practice, simplified models can be used like, in particular, a model consisting of a set of pairs:

$$
M_{\varepsilon}=\left[Q_{\varepsilon}^{(\mu)}\right](13)
$$

where

$$
Q_{\varepsilon}^{(\mu)}=\left[m_{\varepsilon}^{(\mu)}, \delta_{\varepsilon}^{(\mu)}\right]
$$

$m_{\varepsilon}^{(\mu)}, \boldsymbol{\delta}_{\varepsilon}^{(\mu)}$ denote, respectively, the mean value and standard deviation of the $\mu^{\text {th }} M S$ component normalized by division by $v_{S S}$; $\mu$ being standing for the $r^{\text {th }}$ order MS2 component indices. Let, like in Sec 5, $U^{(\kappa, \lambda)}$ be a given $r^{\text {th }}$ order working sub-array and $W_{k, \lambda}$ - its $r^{\text {th }}$ order 2-dimensional MS2 (consisting in this case of $2^{2 r}$ components): 


$$
W_{\kappa, \lambda}=\left[w^{(S S \ldots S)}, w^{(S \ldots \ldots V)}, \ldots, w^{(X X \ldots H)}, w^{(X X \ldots X)}\right] .
$$

Before classification of $U^{(\kappa, \lambda)}$, in order to make it independent of the brightness level, the spectral components should be normalized according to the formula:

$$
z^{(\mu)}=\frac{w^{(\mu)}}{w^{(S \ldots S)}} \cdot(17)
$$

Classification of $U^{(\kappa, \lambda)}$ then can be based on evaluation Euclidean distances between the normalized spectrum $\bar{W}_{e, .}$ and the porosity models $M_{\varepsilon}, \varepsilon=1,2, \ldots, e$, according to the formula:

A decision:

$$
\tilde{n}\left(\bar{W}_{\hat{e}, \bar{c}}, M_{a}\right)=\sqrt{\sum_{\{\mu\}} \frac{\left(z^{(\mu)}-m_{\varepsilon}^{(\mu)}\right)^{2}}{\left(\delta_{\varepsilon}^{(\mu)}\right)^{2}}} .
$$

$$
U^{(\kappa, \lambda)} \in C_{\varepsilon}(19)
$$

is maid if $\tilde{n}\left(\bar{W}_{\hat{e}, \ddot{e}}, M_{\tilde{a}}\right)$ is minimal for a given $C_{\varepsilon^{\prime}}$ Similar decisions made in all working windows of an examined image are a basis of global porosity assessment in a given sample of porous material.

\section{Conclusion}

Computer-aided analysis of microscope images of porous material sections is a typical approach to evaluate basic parameters characterizing the materials' porosity. However, it needs segmentation of individual pores, measuring their geometrical parameters and evaluation of secondary pore characteristics. In this paper an alternative approach is proposed. It is based on partition of the porous material section image into regular (square) working windows, computer calculation of morphological spectra (MS) components of sub-images in an established order working windows and assigning the spectra to previously established similarity classes corresponding to various types of porosity. Such approach spares calculation costs because $M S$ components calculation consists only of adding and/or subtracting pixel values, the number of working windows being also strongly limited. The above-proposed method of porosity evaluation is still in the state of development.

\section{References}

1. Drukker W (1989) Haemodialysis: A historical review. Replacement of renal functions by dialysis, Kluwer Academic Publishers. Dordrecht, Netherlands, pp. 20-86.
2. Chwojnowski A, Wojciechowski C, Dudziński K, Łukowska E, Granicka L (2009) New type of hollow fiber membrane for cell and microorganism's cultivation and encapsulation. Desalination 240(1-3): 9-13.

3. Kinasiewicz A, Dudziński K, Chwojnowski A, Weryński A, Kawiak J (2007) Three-dimensional culture of hepatocytes on spongy polyethersulfone membrane developed for cell transplantation. Transplant Proc 39(9): 2914-2916.

4. Przytulska M, Kulikowski JL, Lewińska D, Grzeczkowicz M, Kupikowska SB (2015) Computer-aided image analysis for microcapsules' quality assessment. Biocybernetics and Biomedical Engineering 35(4): 342-350.

5. Przytulska M, Kruk A, Kulikowski JL, Wojciechowski C, Gadomska GA, et al. (2017) Comparative assessment of polyvinylpyrrolidone type of membranes based on porosity analysis. Desalination and Water Treat 75: $18-25$

6. Pratap R (2006) Getting started with MATLAB 7. Oxford University Press, UK.

7. Piętka D (2017) The MeMo Explorer ${ }^{\mathrm{TM}}$. An advanced membrane morphology software, version 17.10.24.0, User Guide, Inner Rep IBBE PAS, Warsaw, Poland.

8. Russell S, Norvig P (2003) Artificial intelligence. A modern approach, ( $2^{\text {nd }}$ edn), Pearson Education International, Upper Saddle River NJ, USA.

9. Walsh JL (1923) A closed set of orthogonal functions. Am J Math 45(1): 5-24.

10. Lazaridis G, Petrou M (2006) Image registration using the walsh transform. IEEE Trans Image Process 15(8): 2343-2357.

11. Kulikowski JL, Przytulska M, Wierzbicka D (2007) Morphological spectra as tools for texture analysis. In: Kurzynski M (Ed.), Computer Recognition Systems 2. ASC 45, Springer-Verlag, Berlin, Germany, pp. 510-517.

12. Kulikowski JL, Przytulska M, Wierzbicka D (2010) Description of biomedical textures by statistical properties of morphological spectra. Biocybernetics and Biomed Eng 30(3): 19-34.

13. Przytulska M, Kulikowski JL, Wierzbicka D (2011) Discrimination of poorly distinguishable random textures by statistical analysis of morphological spectra. Machine Graphics and Vision 20(4): 455-477.

14. Russ JC (2011) The image processing handbook, (6 $6^{\text {th }}$ edn), CRC Press, Boca Raton, USA. 